\title{
Assessment of a Pediatric Patient
}

Kristine M. Ruggiero

\section{INTRODUCTION}

The history and physical exam (PE) remain a clinician's most important tools. Many times a complete history and PE reveal findings unrelated to and unexpected from the patient's chief complaint and major concerns (Boxes 1.1 and 1.2).

An age-specific approach to the pediatric PE is another important consideration when caring for children. Remember historical information depends almost completely upon the caregiver for the patient, especially in infants and very young children.

\section{OBJECTIVES}

1. To understand the differences in the approach to care of a pediatric patient based on the child's age and developmental level:
a. Prenatal and birth history
b. Developmental history
c. Social history of family
d. Immunization history

2. Overview of how to approach the history and PE of a pediatric patient based on their age and in the context of the parent/caregiver-child relationship during the pediatric healthcare encounter.

\section{KEY POINTS IN OBTAINING THE HISTORY}

Key elements of the history-taking process (Box 1.1) are to establish a warm, caring atmosphere and be nonconfrontational. It is important to greet everyone in the room in a polite manner, with a friendly demeanor. Remember to wash your hands before and after your exam while in the patient's exam room. Getting down to the child's eye level upon entering the room (and not towering over them) is also important, especially when examining toddlers and preschoolers. Distraction is a valuable tool. Be honest. If something is going to hurt, tell the child that in a calm, age-appropriate fashion. Do not lie, or else you will lose credibility. Remember that the birth history and the impact that this has on the child's growth and development are key differences between a pediatric and adult patient (Hagan, Shaw, \& Duncan, 2020). 
- Infants under 6 months of age who tend to not have stranger anxiety are best examined on the exam table.

- For infants older than 6 months and anxious toddlers who are leery of strangers, the exam may be more comfortable if sitting on their parent/caregiver's lap.

- While children over 3 years of age tend to be generally more cooperative during the exam without being held, they may like to play with the equipment beforehand (i.e., allowing patients to hold the stethoscope or otoscope before using it on them).

- The PE of children aged 5 to 12 years old and adolescents are easier to perform because they also tend to cooperate more.

\section{OUTLINE FOR PEDIATRIC HISTORY AND PHYSICAL EXAM}

\section{BOX 1.1 OUTLINE FOR PEDIATRIC HISTORY AND REVIEW OF SYSTEMS}

I. Presenting Complaint (Informant/Reliability of Informant): Document the chief complaint and its duration in the patient's or parent's own words whenever possible.

II. Present Illness: Begin with statements that include age, sex, color, and duration of illness (note source of information and relationship to the patient, i.e., mother, father, grandparent, caregiver).

History of the present illness (HPI): "OLDCART” (acronym for onset, location, duration, character/quality, aggravating/alleviating, relieving factors, timing).

How and when did the illness start?

Health immediately before the illness.

Progress of disease; order and date of onset of new symptoms.

Specific symptoms and physical signs that may have developed.

Any pertinent negative data (i.e., no fever, denies sore throat, etc.).

Aggravating and alleviating factors.

Significant medical attention and medications given, dosages, and over what period and when the last dose was given.

In acute infections, ask about the type and degree of exposure. For the well children, ask how they have been since their last visit. Any concerns?

III. Birth History/Neonatal History

A. Birth History:

1. Prenatal: Did the mother receive prenatal care? Use of prenatal vitamins? Health of mother during pregnancy. Medical supervision, drugs, diet, infections such as rubella, other illnesses, vomiting, toxemia, other complications.

2. Antenatal: Rh typing and serology, maternal bleeding, mother's previous pregnancy history.

3. Natal: Duration of pregnancy, birth weight, kind and duration of labor, type of delivery, presentation, sedation and anesthesia (if known), state of infant at birth, resuscitation required, onset of respiration, first cry. 


\section{BOX 1.1 OUTLINE FOR PEDIATRIC HISTORY AND REVIEW OF SYSTEMS (continued)}

\section{B. Neonatal:}

1. Ask what the Apgar scores were. Sometimes parents/caregivers do not know this information. Another way to ask if there were complications after the baby was born is to ask if the baby went home from the hospital with the mother/parents or stayed in the hospital longer. Also ask if the baby went to the NICU.

2. Ask about any congenital anomalies or birth injuries.

3. Ask about any jaundice, transfusion, sepsis, or other neonatal problems.

4. Ask if the baby had any difficulty in sucking, any rashes, excessive weight loss, or feeding difficulties.

IV. Previous History (Including Past Medical History [PMH], Past Surgical History [PSH], Past Illnesses)

\section{A. Past Illnesses:}

1. First document the child's previous general health (i.e., "Previously healthy 1-yearold male"), and then the specific areas listed below should be explored.

B. PMH (Including all diagnoses, infections, accidents, and injuries [including ingestions]): age, type/nature, severity, sequelae.

C. Past Hospitalizations: Including place of hospitalization, for what reason, and duration of hospitalization. And/or past ED visits, including what hospital, for what reason, and when.

D. Past Surgeries: Where and by whom for what diagnosis.

\section{Vaccination History/Immunizations}

A. List date and type of immunization, facility providing immunization, and any complications or reactions.

B. Do not list: "Up to date per parent report." If no immunization record is available, then note this in the problem section of the plan so this can be followed up on.

VI. Developmental History (Growth and Development)

A. Development: Remember to ask about motor (gross and fine) and social (interaction with others) as well as some major milestones achieved (i.e., age smiled, sat, crawled, walked, first words). Can ask about school performance or comparison with siblings in the older child.

B. Gross motor (e.g., head control, sitting, pulling to stand, walking).

C. Fine motor (e.g., raking, pincer grasp, refined pincer grasp).

D. Social (e.g., social smile, smiling, interaction with toys).

E. Cognitive (e.g., object permanence, school performance).

Note major growth and development milestones for age and if parent/caregiver has any concerns about infant's development (i.e., first raised head, rolled over, sat alone, pulled up, walked; Feigelman, 2018).

Note any formal developmental screening when appropriate and also if infant/child is receiving services for any delay.

(See Chapter 2, Pediatric Growth and Development.)

\section{Nutrition History}

A. Breast or formula: Type, duration, major formula changes, time of weaning, difficulties.

B. Be specific about how much milk or formula the baby receives. How does caretaker mix the formula?

C. Vitamin supplements: Type, when started, amount, duration.

D. "Solid" foods: When introduced, how taken, types.

E. For older child, ask about diet, meals/snacks per day, and types of foods consumed.

F. Appetite: Food likes and dislikes, idiosyncrasies or allergies, reaction of child to eating. An idea of child's usual daily intake is important. 


\section{BOX 1.1 OUTLINE FOR PEDIATRIC HISTORY AND REVIEW OF SYSTEMS (continued)}

VIII. Medications and Allergies

A. Allergies, with specific attention to drug allergies: Detail type of reaction. Results of allergy testing if performed.

B. Medications that patient is currently taking: Prescribed, over the counter (OTC), homeopathic, including dose, formulation, route, and frequency.

IX. Family History (Use Family Tree Whenever Possible)

A. Age and health of family members (parents, grandparents, siblings).

B. Stillbirths, miscarriages, abortions; age at death and cause of death of immediate members of family.

C. Known genetic diseases.

D. Conditions with a genetic contribution: Allergy, blood dyscrasias, mental or nervous diseases, diabetes, cardiovascular diseases, kidney disease, rheumatic fever, neoplastic diseases, congenital abnormalities, cancer, convulsive disorders, and others.

E. Health of contacts: Any recent ill exposures (i.e., TB).

X. Social History

A. Type of habitat, age of habitat, number of people in home and relationship(s) to patient

B. Marital status of parents and involvement with child

C. Parents' employment

D. Child care or school

XI. Environmental History

A. Environmental tobacco smoke

B. Water source to home

C. Pets

D. Smoke and carbon monoxide (CO) detectors

E. Firearms

\section{REVIEW OF SYSTEMS (ROS)}

A system review will serve several purposes:

A. It will often bring out symptoms or signs missed in collection of data about the present illness.

B. It might direct the interviewer into questioning about other systems that have some indirect bearing on the present illness (e.g., eczema in a child with asthma).

C. It serves as a screening device for uncovering symptoms, past or present, which were omitted in the earlier part of the interview.

ROS:

A. General: Unusual weight gain or loss, fatigue, temperature sensitivity, mentality. Pattern of growth (record previous heights and weights on appropriate charts). Time and pattern of puberty (use Tanner Staging when appropriate).

B. Eyes: Have the child's eyes ever been crossed? Any foreign body or infection, glasses for any reason. Any problems with their vision, any eye pain?

C. Ears, Nose, and Throat: Frequent colds, sore throat, sneezing, stuffy nose, discharge, postnasal drip, mouth breathing, snoring, otitis, hearing, adenitis.

D. Teeth: Age of eruption of deciduous and permanent; number at 1 year; comparison with siblings.

E. Cardiorespiratory: Frequency and nature of disturbances. Dyspnea, chest pain, cough, sputum, wheeze, expectoration, cyanosis, edema, syncope, tachycardia.

F. Gastrointestinal: Vomiting, diarrhea, constipation, type of stools, abdominal pain or discomfort, jaundice. 


\section{BOX 1.1 OUTLINE FOR PEDIATRIC HISTORY AND REVIEW OF SYSTEMS (continued)}

G. Genitourinary: Enuresis, dysuria, frequency, polyuria, pyuria, hematuria, character of stream, vaginal discharge, menstrual history, bladder control, abnormalities of penis or testes. Details of menarche and menstruation for adolescent females.

H. Neuromuscular: Headache, nervousness, dizziness, tingling, convulsions, habit spasms, ataxia, muscle or joint pains, postural deformities, exercise tolerance, gait.

I. Endocrine: Disturbances of growth, excessive fluid intake, polyphagia, goiter, thyroid disease.

J. Hematologic: Bruise easily, difficulty stopping bleeds, lumps under arms, neck; fevers, shakes, shivers.

K. Rheumatologic: Joints: pain, stiffness, swollen, variation in joint pain during day, fingers painful/blue in cold, dry mouth, red eyes, back, neck pain.

L. Skin: Ask about rashes, hives, problems with hair, skin texture or color, and the like.

There are differences in performing a pediatric physical examination compared with that of an adult, and it is important to gather as much data as possible. There is an agreed-upon approach to evaluation of a child. The following three recommendations will aid in the evaluation of the child. First, stay at the child's level while observing them; second, get as much history from them as possible; and third, do the exam from least distressing to most distressing.

\section{BOX 1.2 AGE-SPECIFIC APPROACH TO THE PHYSICAL EXAM}

Infant: Lying in parent/caregiver's arms, leave eyes, ears, mouth to end (least to most invasive approach), do heart/lungs when infant is quiet or sleeping, try distraction techniques with older infants.

Toddler: Minimal contact, allow to inspect equipment (i.e., stethoscope), assess heart/lungs and then head-to-toe approach.

Preschooler: Similar to the toddler, especially allow the preschooler to handle the equipment.

School Age: Respect privacy, explain steps/approach to PE beforehand.

Adolescent: Explain findings; same as school-age child, respect privacy; ask parents to leave room during part of the exam.

\section{References}

Feigelman, S. (2018). Developmental and behavioral theories. In Nelson's textbook of pediatrics (21st ed., pp. 117-123). Philadelphia, PA: Elsevier.

Hagan, J., Shaw, J., \& Duncan, P. (2020). Bright futures: Guidelines for health supervision of infants, children and adolescents (4th ed.). [ebook]. https://doi.org/aappublications.org/ bright-futures-guidelines 